\title{
Reduced magnesium intake and elevated predisposition to cardiovascular disorders: a systematic review
}

\author{
B. Dasbulak ${ }^{1}$, S. C. Langley-Evans ${ }^{1}$ and S. J. M. Welham ${ }^{1}$ \\ ${ }^{1}$ Division of Nutritional Sciences, University of Nottingham, Sutton Bonington, Loughborough, \\ Leicestershire, LE125RD, UK
}

Magnesium (Mg) is an essential mineral for human health and acts as a cofactor for more than 325 enzymes ${ }^{(1)}$. Magnesium status is dependent on level of intake, daily losses (urine, faeces and sweat), malabsorption syndromes, alcohol abuse, and acute vomiting or diarrhoea. Therefore, daily magnesium intake is required to maintain optimal levels ${ }^{(2)}$. The National Diet and Nutrition Survey (NDNS) rolling programme has indicated that Mg intakes are below the Reference Nutrient Intake for both men (RNI - $300 \mathrm{mg} /$ day) and women (RNI - $270 \mathrm{mg} /$ day) in the UK ${ }^{(3)}$. In the UK

population, adolescent girls $(48 \%)$ and women aged $10-64$ years $(11 \%)$ are particularly at risk of dietary deficiency and $16 \%$ of the over 65 s consume magnesium under the LRNI.

While many studies have examined the association between hypomagnesemia and cardiovascular disease, few have been exclusively centred around $\mathrm{Mg}$ intake. Therefore, this systematic review aimed to investigate the relationship between magnesium disorders (deficiency/intake, hypomagnesemia) and risk of cardiovascular diseases (CVD).

We set out to conduct an unbiased systematic review to investigate the association between $\mathrm{Mg}$ intake and risk of cardiovascular diseases around the world.

This systematic review was conducted according to PRISMA (Preferred Items for Systematic Reviews and Meta-Analysis) guidelines. PubMed and Web of Science electronic databases were searched for articles that investigated the association between varying magnesium intake and cardiovascular disorders and death. Only studies published in last 10 years, were included in the study. Incidence of disease and deaths for individuals from low $\mathrm{Mg}(<2.0 \mathrm{mg} \mathrm{dL}-1)$ and high $\mathrm{Mg}(>2.0 \mathrm{mg}$ dL-1) status were used to calculate hazard ratios (HR).

After an initial search yielding 2024 studies, filtering resulted in 8 studies being included in the analysis which incorporated data from $>55000$ participants and included approximately 20000 cases and deaths. The mean HR of the 8 studies was $>1.50$ for the low $\mathrm{Mg}$ group and 1.05 for the high $\mathrm{Mg}$ group for death from CVD. The cardiovascular death rate in hypomagnesemic individuals was $>40 \%$, being higher than for any other cause of death.

$\mathrm{Mg}$ intake is insufficient in developed and developing countries and is directly associated with death from CVD. Elevation of Mg intake or bioavailability in foods may play a significant role in diminishing the incidence of and death from CVD. Solutions ranging from modification of food processing to diminish $\mathrm{Mg}$ losses and water quality management may significantly enhance intakes and diminish this relative risk.

\section{References}

1. Kostov K, Halacheva L. (2018) Int J Mol Sci. 2018;19(6):1-23.

2. Belin RJ, He K. (2007) Magnes Res. 2007;20(2):107-29.

3. Roberts C, Steer T, Maplethorpe N, Cox L, Meadows S, Nicholson S, et al. (2018) NDNS (2014/2015-2015/2016). Public Heal Engl. 2018; 8. 